\title{
Full metal jacket or the emperor's new clothes?
}

Launched with little more than a whimper during the Labour Party Conference the much vaunted National Service Framework for Mental Health (NSF-MH) outlines the Government's 'ambitious agenda' for mental health services (Department of Health, 1999a). The official driving force has been the desire to deliver a quality service throughout the whole NHS via clinical governance and underpinned by professional self-regulation. Developed following widespread consultation and with the advice of the External Reference Group (although some of this advice was clearly disregarded), the NSF-MH provides a series of seven core standards with examples of good practice. Although developed with general psychiatry and severe mental illness in mind, the NSF is not quite the 'National Schizophrenia Framework' that some envisaged, since it also acknowledges the needs of young people and the influence of developmental factors on adult mental health. The NSF-MH sets standards in five areas: mental health promotion, primary care and access to services, services for the severely mentally ill, caring about carers and preventing suicide. It is only the second to be published (the other being for coronary care) which is hopefully a reflection of the 'priority' once more being given to mental health. However, the nearsimultaneous appointment of a cancer 'tsar' suggests that 'priority' is a readily used and easily diluted term.

The principles on which the framework is founded should be commended. We would all agree that we wish to see services that are free of discrimination, accessible to users, offer choices, deliver continuity of care, are well coordinated and are accountable to the public. The attention given to public safety is also essential if we are to rebuild public confidence and avoid the mistakes of the past (e.g. Daily Express, 16 January 1996; Daily Mirror, 9 February 1996). The sensible concern to empower and support staff is certainly needed to raise morale and to stop the drift away from the beleaguered trenches of general adult psychiatry (Deahl \& Turner, 1997). Of course, some may consider these principles to be only a repetition of the standard platitudes doled out in numerous policy documents, so obvious and unarguable as to consider them demeaning to those to whom they are addressed. To be told the NSF-MH "puts in place underpinning programmes to support local delivery" is hardly inspiring and reeks of redundant phraseology.

The drive to ensure that treatments delivered take account of evidence-based research should improve quality and reduce unacceptable variations. Greater choice and standards of care in different parts of the UK will, however, require much greater integration of health, social services and the voluntary sector than at present. These alliances have already demonstrated their effectiveness particularly in inner city areas (Hadley \&
Goldman, 1995; Strathdee \& Thornicroft, 1996), but remain at risk if single gender wards, constant reconfigurations, fragmented funding arrangements and interagency rivalries persist. Perhaps most importantly the recent calls for what patients and their carers want from services have been acknowledged, and should now routinely be incorporated into the planning and delivery of care (Noble et al, 1999).

\section{Mental health promotion}

The first standard relates to mental health promotion. Local services will be required to develop a health improvement plan "demonstrating action within and linkages between organisations", that targets vulnerable groups so as to try to prevent mental illness. While this is commendable it seems hard to envisage how community teams, who are already overstretched by the needs of the severely mentally ill (Fulop et al, 1996), could devote much time to this without a large injection of resources. Are psychiatrists to become responsible for providing cognitive-behavioural training to all the unemployed in their catchment areas simply because there is evidencebased research which shows that there are interventions that can reduce the psychological impact of job loss (Proudfoot \& Carson, 1997)? While local services are already involved in the care of people with drug and alcohol problems, the inclusion of new formalised responsibilities for the homeless and people in prison, to be managed within existing resources, is wholly unrealistic. The danger of adopting such an ambitious standard is that it puts limitless demands on local services, they will probably fail, and further blame and demoralisation will ensue.

Mental health services have a duty to inform and educate local communities, but realistically they cannot, nor should they, shoulder the sole responsibility for implementing these interventions. Health education in general has not been particularly successful, and the evidence that such didactic approaches can reduce rates of mental illness simply does not exist. Furthermore, in espousing community initiatives, the boundaries of general psychiatry have to be clearly defined. Local attempts to improve the mental health of whole communities and vulnerable individuals will be unlikely to succeed unless there is a reduction in stigma, and unless new partnerships and ways of working are developed between mental health services and other agencies. Second, if the Government is acknowledging that there are important social causes of psychiatric illness, it too must accept responsibility and continue to address those areas of policy which reduce social exclusion. Social policy 
is largely driven by political ideology and perhaps psychiatrists should be doing more to inform governments and the public about the impact of policy on the nation's mental health? For example, if suicide rates are going to be a model of performance assessment, what about putting methionine in paracetamol tablets or catalytic converters on all car exhausts?

The second part of this standard, which aims to combat the discrimination associated with mental illness, embodies the spirit of the College's 'Changing Minds Every Family in the Land' campaign to reduce stigma and prejudice against patients and their carers, and is very welcome. Psychiatrists are as stigmatised as their patients, both within the medical profession and among the public, and no other medical speciality has the potential to be viewed with such suspicion and hostility. Psychiatrists can only educate society if they are taken seriously as a profession so as to inspire public trust and confidence. We must work to improve our image and professional self-esteem if we are not to risk losing influence in generating policy and implementing the NSF reforms. There is a ' $10 \mathrm{am}-4 \mathrm{pm}$ ' image of staid inflexibility in many areas of psychiatric practice, and general practitioners (GPs) are understandably frustrated at the protocol-bound, time-limited, catchment-area defined, sub-speciality demarcated procedures which they often face.

\section{Primary care and access to services}

The next standard concerns primary care and is an important recognition of the need for quality assessment by GPs of patients' mental health and the recognition of common disorders like depression and anxiety. It encourages GPs to become more familiar with psychological treatments and to improve communication skills. Although this would seem a good idea, it will doubtless result in more referrals to specialist services. This will have to be met by an increase in resources for specialist psychotherapy services if waiting lists are not to get even longer, and is likely to lead to increasing dissatisfaction of the public and our GP colleagues. GPs themselves will find it extremely difficult to create the time needed for such assessments in the melee of overcrowded clinics (Murray et al, 1996). The real dilemma for them is how to keep people out of their surgeries!

The third standard, which aims to give patients 24 hour access to 'adequate' care (whatever that means) from their local service, in the form of the multidisciplinary mental health team, will be problematic and expensive to execute. Keyworkers cannot be available 24 hours a day, 365 days a year. The pay and conditions of service of commmunity psychiatric nurses (CPNs) and health care support workers are lamentable, and there are already serious staffing shortfalls in many community mental health teams (CMHTs). If mental health clinicians are to be available in the evenings and weekends their work must be made professionally rewarding and the expense of unsociable hours payments will have to be met. The traditional backstop of mental health services, the duty hospital senior house officer, cannot be allowed to shoulder the heightened public expectation of this new 'open all hours' approach.

Perhaps 24-hour helplines will be able to share some of the burden of need, but organisations like the Samaritans and SANE are staffed by skilled unpaid volunteers and have no contract of care with callers, such as would be ensured by a line set up by local mental health services. The latter would be expensive, as it would presumably be manned by mental health professionals. It would also seem difficult for such a service to be able to respond to a diverse range of cultural needs, particularly in inner cities, 24 hours a day. If new services are established to respond to need around the clock, care must be taken to ensure continuity and effective liaison with $\mathrm{CMHTs}$, the keyworker and the responsible medical officer (RMO). New services create additional boundaries between which patients have a habit of slipping, even if they are redesignated as 'service users'. Helplines may seem appealing, but have no direct responsibility, and there is no evidence that suicidal acts are reduced by such measures (Evans et al, 1999).

\section{Effective services for people with severe mental illness}

The management of the severely mentally ill using a simplified two-tier Care Programme Approach (CPA) is embraced by the fourth standard. For a framework that prides itself on being evidence-based this would appear a contradiction in terms, as the bureaucracy of the CPA was introduced without evidence of benefit. Indeed, there is mounting evidence that the CPA may be ineffective and does not justify the considerable resources needed for its implementation (Holloway \& Carson, 1998; Tyrer, 1998; UK700 Group, 1999). Patients are meant to receive care that "optimises engagement", with a written care plan, regularly reviewed by a "care co-ordinator". This linguistic gobbledegook is little more than patronising common sense, and is to be measured by a reduced suicide and readmission rate (among other vaguer measures). So if brief crisis admissions are allowed (which many patients and carers want) you will be seen to be failing in "care optimisation"!

Predicting crises with the most sophisticated risk assessment protocols is difficult in a group who are often hard to engage and non-adherent with medication. The lack of suitable accommodation near local services often means people are placed out of area and then become the responsibility of services that do not know the extent of their problems, the classic example being the case of Christopher Clunis (Ritchie, 1994). Poor interagency communication and the burden of workloads can mean patients are still lost to follow-up. The emphasis on written care plans, though well meaning, remains at heart a bureaucratic process that may reduce face-to-face contact with patients, and also generates a mountain of paperwork. When crises do occur the lack of available inpatient beds, especially in London (Audini et al, 1999), will make standard five unachievable until there are either 
more beds or the development of appropriately staffed alternatives.

Acute psychiatric beds are still viewed with suspicion by planners who continue to ignore rising Mental Health Act section rates (Department of Health, 1999b) and who believe that significant reductions in bed numbers and cost savings can be made if effective community services are in place. Care will have to be taken to resist the temptation to close beds in an attempt to be seen to implement NSF-MH standards in the community. Reorganisation of these crowded hospital wards to meet the Government's commitment to single gender facilities is also likely to increase pressures in the short term, while the lack of supported 24-hour accommodation continues to block beds. Resisting the temptation to implement standards before there are services that are adequately resourced to support them may prove difficult. Yet all the pressure generated by inquiry reports, risk management protocols and intensive CPA ends up by forcing clinicians into early admission and delayed discharge.

\section{Caring about carers}

The sixth standard focuses on the needs of carers and formalises what was probably already being done on an ad hoc basis by many community teams. There will now be an obligation to assess carers formally at least once a year and for them to have their own written care plan. It is arguable whether such a formal assessment of a carer's needs is required, or even welcome, if it diverts resources away from the very people for whom they care. There also needs to be clarification of exactly what 'substantial care' means, while the knotty issue of patient confidentiality remains an unresolved dilemma.

\section{Preventing suicide}

One of the ways the Government will assess the implementation of the standards is by using the suicide rate, and strategies to prevent suicide are outlined in the seventh and final standards. In Saving Lives - Our Healthier Nation (Department of Health, 1998) a commitment was made to reduce the suicide rate by at least one-fifth by 2010. The belief is that by implementing the other six standards, as well as by improving the training of staff, auditing procedures and building links between prisons and courts, health and social services, it will be possible to achieve this target. However, rates of suicide are more closely linked to employment rates, gender, age and occupation than to levels of mental illness or the effectiveness of mental health services (Gunnell \& Frankel 1994; Lewis \& Sloggett, 1998).

Although a reduction in suicides is a worthy objective its value as a performance measure of local services, and how they are reaching the standards in the framework, is more than questionable. Of the high numbers of young men committing suicide very few have contact with any medical or psychiatric services, many are transient and whether or not they will use NHS Direct or other tele- phone helplines is equally uncertain (Matthews et al, 1994).

\section{Discussion}

There is a general expectation that many of the standards can be achieved within existing resources, yet the extra responsibilities for carers, closer liaison with prisons, 24hour assertive outreach teams, 24-hour helplines and special services for vulnerable groups will all need extra funds. It is not clear whether that which is promised ( $f 700$ million over three years) will be linked to evidencebased research, or what extra resources are needed to achieve the standards. Although this seems a substantial sum it is little compared with the $₫ 32$ billion mental illness is believed to cost the country each year (Patel \& Knapp, 1998). The NSF-MH acknowledges the problems of recruitment and retention of staff, but offers no strategic solutions to an issue that needs to be addressed if its standards are to be a success. It is ironic that while acknowledging the burden on family and informal carers there is no mention of the burdens facing professional carers (Lancet, 1994; Prosser et al, 1996). Perhaps if psychiatrists and other mental health workers were subject to their own annual needs assessment morale, recruitment and retention might benefit as a result? Furthermore, by emphasising the tasks to be 'kitemarked' at a local level there is a subtle shift of responsibility away from those operating in national and regional offices.

Beleaguered in recent years, general adult psychiatry remains the cornerstone of the nation's mental health services. We consider that it is a rewarding and immensely satisfying area of practice. By education or direct intervention it offers a unique opportunity significantly to improve the lives of the one-sixth of the population who, according to the NSF-MH, suffer from mental illness at any one time. The NSF-MH is a mandate for general psychiatry, which if implemented, adequately resourced and underpinned by sensible reforms of mental health legislation could go a long way to restore confidence and morale within the adult mental health services. It might also offer some protection to psychiatric services if they end up being managed within primary care trusts. In our view, training in psychiatry and mental health nursing must evolve to meet the challenges created by the NSF-MH.

General psychiatrists may, however, be forgiven for a touch of scepticism, surrounded as we now are by NSF$\mathrm{MH}$ newspeak. Many fine words, government guidance and legislation over the last decade have done little to improve the everyday difficulties - especially in inner cities. The NSF-MH is somewhat 'motherhood and applepie', bland and often lacking in detail. It is wishy-washy about getting rid of health/social service boundaries that so constantly fragment coordinated practice. It offers little guidance on how standards should be implemented, delegating that formidable task to a number of 'implementation' groups. 
opinion \& debate
We hope the NSF-MH will be properly 'implemented', but wonder whether its 'kite-mark' words will be matched with effective actions. It offers some brave (and some would say rash) commitments which the Government may yet live to regret, for example, making psychological therapies available for all. This is laudable but has dangerous implications. For by widening the goalposts of service provision it is all too easy (as history tells us) to be diverted from treating the seriously mentally ill. We must also not allow the boundaries of general psychiatry to be broadened to absurdity by government attempts to divert blame and to medicalise matters of social policy. The Cinderella service has seen many a fancy ballgown offered by its various fairy godpersons, but calling its new dress an 'innovative imperial vestment', or even a 'robust prioritised framework', will not prevent the mockery and disappointment that goes with the ill-fitting glass slipper of historic under-resourcing.

\section{References}

AUDINI, B., DUFFETT, R., LELLIOT, P., et al (1999) Over-occupancy in London's acute psychiatric units - fact or fiction? Psychiatric Bulletin, 23, 590-594.

DEAHL, M. \& TURNER, T. (1997) Genera psychiatry in no-man's land. British Journal of Psychiatry, 171, 6-8.

DEPARTMENT OFHEALTH (1998) Saving Lives - Our Healthier Nation. A Contract for Health. A Consultation Paper. London: Department of Health.

- (1999a) A National Service Framework for Mental Health. London. Department of Health.
— (1999b) In-patients Formally Detained in Hospitals under the Mental Health Act 1983 and Other Legislation, England: 1988-89 to 1998-99. Statistical Bulletin 1999/25. London: Department of Health.

EVANS, M O, MORGAN, H. G. HAYWARD, A., et al (1999) Crisis telephone consultation for deliberate self-harm patients: effects on repetition. British Journal of Psychiatry, $175,23-27$

FULOP, N. J., KOFFMAN, J., CARSON, $\mathrm{S}$. et al (1996) Use of acute psychiatric beds: a point prevalence survey in North and SouthThames regions. Journal of Public Health Medicine, 18 207-216
GUNNELL, D. \& FRANKEL, S. (1994) Prevention of suicide: aspirations and evidence. British Medical Journal, 308, 1227-1233.

HADLEY,T. R. \& GOLDMAN, H. (1995) Effects of recent mental health and social service policy reforms on Britain's mental health system. British Medical Journal, 311, 1556-1558.

HOLLOWAY, F. \& CARSON, J. (1998) Intensive case management for the severely mentally ill. Controlled trial. British Journal of Psychiatry, 172, 19-22.

LANCET (1994) Editorial: Burnished or burnt-out: the delights and dangers of working in health. Lancet, 44 1583-1584

LEWIS, G. \& SLOGGETT, A. (1998)

Suicide, deprivation, and unemployment: record linkage study. British Medical Journal, 317, 1283-1286.

MATHEWS, K., MILNE, S. \& ASHCROFT, G.W. (1994) Role of doctors in the prevention of suicide: the final consultation. British Journal of General Practice, 44, 345-348.

MURRAY, V.,WALKER, H.W., MITCHELL, C., et al (1996) Needs for care from a demand led community psychiatric service: a study of patients with major mental illness. British Medical Journal, 312, 1582-1586

NOBLE, L. M., DOUGLAS, B. C. \& NEWMAN S. P. (1999) What do patients want and what do we know? A review of patients' requests of psychiatric services. Acta Psychiatrica Scandinavica, 100, 321-327.

PATEL, A. \& KNAPP, M. (1998) Costs of mental illness in England. PRRSU Mental Health Research Review, 5, 4-10.

PROSSER, D., JOHNSON, S., KUIPERS, E., et al (1996) Mental health, 'burnout', and job satisfaction among hospital and community-based mental health staff.

British Journal of Psychiatry, 169 334-337.

PROUDFOOT, J. \& CARSON, J. (1997) Effect of cognitive behavioural training on job finding among long-term unemployed people. Lancet, 350 96-100.

RITCHIE, J. H. (1994) The Report of the Inquiry into the Care and Treatment of Christopher Clunis. London: HMSO.

STRATHDEE, G. \& THORNICROFT, G. (1996) Commissioning Mental Health Services. London: HMSO.

TYRER, P. (1998) Cost-effective or profligate community psychiatry? British Journal of Psychiatry, 172,1-3.

UK700 GROUP (1999) Intensive versus standard case management for severe psychotic illness: a randomised trial. Lancet, 353, 185-189.

* Martin Deahl Consultant Psychiatrist and Senior Lecturer in Psychological Medicine, Director of Postgraduate Education, Brian Douglas Specialist Registrar, TrevorTurner Consultant Psychiatrist and Honorary Senior Lecturer in Psychological Medicine, Division of Psychiatry, EastWing, 2nd Floor, Homerton Hospital, Homerton Row, London E9 6SR 